\title{
A Comparison of Approximate Interval Estimators for the Bernoulli Parameter
}

\author{
Lawrence M. LeEMIS and Kishor S. TRIVEDI
}

We compare the accuracy of two approximate confidence interval estimators for the Bernoulli parameter $p$. The approximate confidence intervals are based on the normal and Poisson approximations to the binomial distribution. Charts are given to indicate which approximation is appropriate for certain sample sizes and point estimators.

KEY WORDS: Binomial distribution; Confidence interval; Poisson distribution.

Lawrence M. Leemis is Associate Professor, Department of Mathematics, The College of William \& Mary, Williamsburg, VA 23187-8795. Kishor S. Trivedi is Professor, Department of Electrical Engineering, Duke University, Box 90291, Durham, NC 27708-0291. This research was supported by the National Aeronautics and Space Administration under NASA Contract NAS1-19480 while the authors were in residence at the Institute for Computer Applications in Science and Engineering (ICASE), NASA Langley Research Center, Hampton, VA. Their support is gratefully acknowledged. Helpful comments from Pam Burch, Herb Multhaup, Bruce Schmeiser, Laura Vignati, the editor, associate editor, and two anonymous referees are also acknowledged. 


\section{INTRODUCTION}

The normal approximation to the binomial distribution is appropriate when $n$ is large and $p \approx \frac{1}{2}$. There is conflicting advice concerning which values of $n$ and $p$ are appropriate for using the approximation. For example, a sampling of textbooks recommends that the normal distribution be used to approximate the binomial distribution when:

- $n p$ and $n(1-p)$ are both greater than 5 (Aczel 1993, p. 211; Anderson, Sweeney, and Williams 1994, p. 218; Creighton 1994, p. 159; Freund 1992, p. 245; Goldman and Weinberg 1985, p. 304; Jarrell 1994, p. 280; Kelly 1994, p. 375; Lapin 1990, p. 185; Mason, Lind, and Marchal 1994, p. 251; Triola 1995, p. 266; Trivedi 1982, p. 497; Walpole and Myers 1993, p. 161; Weiss 1995, p. 392)

- $p \pm 2 \sqrt{\frac{p(1-p)}{n}}$ lies in the interval $(0,1)$ (Scheaffer and McClave 1990, p. 242; Mendenhall and Sincich 1992, p. 299)

- $n>9 \max \left\{\frac{1-p}{p}, \frac{p}{1-p}\right\}$ (Larson 1995, p. 197)

- $n p(1-p) \geq 10($ Ross 1994, p. 219)

- $n p(1-p)>9$ (Aczel 1993, p. 158).

Many other textbook authors give no specific advice concerning when to use the normal approximation. To complicate matters further, most of this advice concerns using these approximations to compute probabilities. Whether these same rules of thumb apply to confidence intervals is seldom addressed. The Poisson approximation, although less popular than the normal approximation to the binomial, is useful for large values of $n$ and small values of $p$. The same sampling of textbooks recommends that the Poisson distribution be used to approximate the binomial distribution when $n \geq 20$ and $p \leq 0.05, n \geq 100$ and $p \leq 0.05$, or $n \geq 100$ and $n p \leq 10$ (Creighton 
1994, p. 115; Hogg and Tanis 1993, p. 177; Freund 1992, p. 204; Larson 1995, p. 157; Weiss 1995, p. 321).

Let $X_{1}, X_{2}, \ldots, X_{n}$ be iid Bernoulli random variables with unknown parameter $p$, and let $Y=\sum_{i=1}^{n} X_{i}$ be a binomial random variable with parameters $n$ and $p$. The maximum likelihood estimator for $p$ is $\hat{p}=\frac{Y}{n}$, which is unbiased and consistent. The interest here is in confidence interval estimators for $p$. In particular, we want to compare the approximate confidence interval estimators based on the normal and Poisson approximations to the binomial distribution. Determining a confidence interval for $p$ when the sample size is large using approximate methods is often needed in simulations with a large number of replications and in polling.

Computing probabilities using the normal and Poisson approximations is not considered here because work has been done on this problem. Poor (1991) compared the probability mass functions and cumulative distribution functions of the binomial and associated Poisson approximations with means $n p$ and $-n \log (1-p)$. Ling (1992) suggested using a relationship between the cumulative distribution functions of the binomial and $F$ distributions to compute binomial probabilities. Schader and Schmid (1989) compared the maximum absolute error in computing the cumulative distribution function for the binomial distribution using the normal approximation with a continuity correction. They consider the two rules for determining whether the approximation should be used: $n p$ and $n(1-p)$ are both greater than 5 , and $n p(1-p)>9$. Their conclusion is that the relationship between the maximum absolute error and $p$ is approximately linear when considering the smallest possible sample sizes to satisfy the rules. Azzam, Halawa, and Hussien (1991) considered the same problem, and give a chart indicating whether the normal or Poisson approximation should be used for various $(n, p)$ pairs.

Concerning work done on confidence intervals for $p$, Clopper and Pearson (1934) appear to be the first authors who gave tables, in graphical form, of the confidence 
limits for $p$. Blyth and Still (1983) developed a table of confidence limits for $p$, and gave a correction factor for approximate intervals. Blyth (1986) compared five confidence intervals for $p$. In addition, he used the $F$ distribution to reduce the amount of time necessary to compute an exact confidence interval. Vollset (1993) compared 13 confidence intervals for $p$. Using an arcsin transformation to improve the confidence limits associated with the normal approximation was considered by Chen (1990) and others. Copas (1992) considered a Bayesian confidence interval for p. Daly (1992) gave SAS macros for computing many of the confidence intervals described in this article. Ghosh (1979) compared two confidence intervals for the Bernoulli parameter based on the normal approximation to the binomial distribution.

\section{CONFIDENCE INTERVAL ESTIMATORS FOR $p$}

Two-sided confidence interval estimators for $p$ can be determined with the aid of numerical methods. One-sided confidence interval estimators are analogous. Let $p_{L}<p<p_{U}$ be an "exact" (Blyth 1986) confidence interval for $p$, where $p_{L}$ and $p_{U}$ are functions of the sample size $n$, the number of successes $y$, and the stated coverage of the interval $1-\alpha$. For $y=1,2, \ldots, n-1$ the lower limit $p_{L}$ satisfies (see, for example, Larsen and Marx 1986, p. 279)

$$
\sum_{k=y}^{n}\left(\begin{array}{c}
n \\
k
\end{array}\right) p_{L}^{k}\left(1-p_{L}\right)^{n-k}=\alpha / 2
$$

For $y=1,2, \ldots, n-1$, the upper limit $p_{U}$ satisfies

$$
\sum_{k=0}^{y}\left(\begin{array}{c}
n \\
k
\end{array}\right) p_{U}^{k}\left(1-p_{U}\right)^{n-k}=\alpha / 2 .
$$

The $\left(p_{L}, p_{U}\right)$ pairs were tabled in Lentner (1982) for $\alpha=0.01$ and $\alpha=0.05$ and $n=2,3, \ldots, 100$, as well as a discussion of the $y=0$ and $y=n$ cases. This confidence interval requires numerical methods to determine $p_{L}$ and $p_{U}$ and takes longer to calculate as $n$ increases. This interval will be used as a basis to check the 
approximate bounds reviewed later in this section. Figures showing the coverage probabilities for bounds of this type for $n=5$ and $n=10$ were given in Blyth (1986). Following a derivation similar to his (given in Appendix A, along with a similar proof using integration by parts), a faster way to determine the lower and upper limits can be determined. An "exact" confidence interval for $p$ based on the $F$ distribution is

$$
\frac{1}{1+\frac{n-y+1}{y F_{2 y, 2(n-y+1), 1-\alpha / 2}}}<p<\frac{1}{1+\frac{n-y}{(y+1) F_{2(y+1), 2(n-y), \alpha / 2}}}
$$

where the third subscript on $F$ refers to the right-hand tail probability. These confidence limits are identical to the $p_{L}$ and $p_{U}$ limits determined using the binomial distribution. The next paragraph discusses numerical issues associated with determining these bounds.

Mathematica (Wolfram 1991) code for solving the binomial equations numerically for a given $n, y$, and $\alpha$ is given in Appendix $\mathrm{B}$. This code works well for small and moderate sized values of $n$. Some numerical instability occurred for larger values of $n$, so the well-known relationship (Larsen and Marx 1986, p. 101) between the successive values of the probability mass function $f(x)$ of the binomial distribution

$$
f(x)=\frac{(n-x+1) p}{x(1-p)} f(x-1) \quad x=1,2, \ldots, n
$$

was used to calculate the binomial cumulative distribution function. The Mathematica code for determining $p_{L}$ and $p_{U}$ using the $F$ distribution is given in Appendix $\mathrm{C}$. This method is significantly faster than the approach using the binomial distribution, but may encounter numerical difficulty with determining the $F$ ratio percentiles for large degrees of freedom.

The first approximate confidence interval is based on the normal approximation to the binomial distribution. The random variable $\frac{Y-n p}{\sqrt{n p(1-p)}}$ is asymptotically standard normal. Thus an approximate confidence interval for $p$ is

$$
\frac{y}{n}-z_{\alpha / 2} \sqrt{\frac{\frac{y}{n}\left(1-\frac{y}{n}\right)}{n}}<p<\frac{y}{n}+z_{\alpha / 2} \sqrt{\frac{\frac{y}{n}\left(1-\frac{y}{n}\right)}{n}}
$$


when $y=1,2, \ldots, n-1$, where $z_{\alpha / 2}$ is the $1-\alpha / 2$ percentile of the standard normal distribution. This approximation works best when $p=\frac{1}{2}$ (e.g., political polls). It allows confidence limits that fall outside the interval $[0,1]$. Using results from Lehmann and Loh (1990), Chen (1990) considered coverage probabilities of this confidence interval. The arcsin transformation, yielding the interval

$$
|\arcsin (\sqrt{p})-\arcsin (\sqrt{y / n})| \leq \frac{z_{\alpha / 2}}{2 \sqrt{n}}
$$

on $(0,1)$ is known to accelerate the rate of convergence of the normal approximation. He concludes that the arcsin transformation is nearly the optimal transformation in terms of this convergence.

The second approximate confidence interval is based on the Poisson approximation to the binomial (see, for example, Trivedi 1982, p. 498). This confidence interval does not appear in textbooks as often as the first approximate confidence interval. The random variable $Y$ is asymptotically Poisson with parameter $n p$. Therefore the exact lower bound $p_{L}$ satisfying

$$
\sum_{k=y}^{n}\left(\begin{array}{c}
n \\
k
\end{array}\right) p_{L}^{k}\left(1-p_{L}\right)^{n-k}=\alpha / 2
$$

can be approximated with a Poisson lower limit $p_{P L}$ that satisfies

$$
\sum_{k=y}^{\infty} \frac{\left(n p_{P L}\right)^{k} e^{-n p_{P L}}}{k !}=\alpha / 2
$$

or

$$
1-\sum_{k=0}^{y-1} \frac{\left(n p_{P L}\right)^{k} e^{-n p_{P L}}}{k !}=\alpha / 2 .
$$

The left-hand side of this equation is the cumulative distribution function for an Erlang random variable with scale parameter $n p_{P L}$ and shape parameter $y$ (denoted by $\left.E_{n p_{P L}, y}\right)$ evaluated at one. Consequently,

$$
P\left(E_{n p_{P L}, y} \leq 1\right)=\alpha / 2 \text {. }
$$


Since $2 n p_{P L} E_{n p_{P L}, y}$ is a $\chi^{2}$ random variable with $2 y$ degrees of freedom, this reduces to

$$
P\left(\chi_{2 y}^{2} \leq 2 n p_{P L}\right)=\alpha / 2
$$

or

$$
p_{P L}=\frac{1}{2 n} \chi_{2 y, 1-\alpha / 2}^{2}
$$

By a similar line of reasoning, the upper limit based on the Poisson approximation to the binomial distribution is

$$
p_{P U}=\frac{1}{2 n} \chi_{2(y+1), \alpha / 2}^{2} .
$$

This approximation works best when $p$ is small. It would be appropriate, for example, in a Monte Carlo analysis of a model of a computer's reliability. If the probability of failure is small and the number of replications is large, the Poisson approximation performs better than the normal approximation.

Examples: Using $n=10, y=3$, and $\alpha=0.05$, the intervals described in this section are:

\begin{tabular}{l|c|c} 
Method & Lower Bound & Upper Bound \\
\hline Exact & 0.067 & 0.652 \\
Normal approximation & 0.016 & 0.584 \\
Arcsin transformation & 0.071 & 0.603 \\
Poisson approximation & 0.062 & 0.877
\end{tabular}

Using $n=100, y=3$, and $\alpha=0.05$, the intervals described in this section are:

\begin{tabular}{l|c|c} 
Method & Lower Bound & Upper Bound \\
\hline Exact & 0.0062 & 0.085 \\
Normal approximation & -0.0034 & 0.063 \\
Arcsin transformation & 0.0058 & 0.072 \\
Poisson approximation & 0.0062 & 0.088
\end{tabular}

The normal approximation is superior to the Poisson approximation in terms of maximum absolute error when $p$ is close to $\frac{1}{2}$, the Poisson approximation is superior to the normal approximation when $p$ is close to 0 , and the arcsin transformation accelerates the convergence of the normal approximation. 


\section{COMPARISON OF THE APPROXIMATE METHODS}

There are several different ways to compare the approximate confidence interval limits with the exact values. These ways include considering the differences between the upper and lower bound errors individually, and considering relative errors. We have decided to compute the error of an approximate two-sided confidence interval as the maximum absolute error

$$
\max \left\{\left|p_{L}-\tilde{p}_{L}\right|,\left|p_{U}-\tilde{p}_{U}\right|\right\}
$$

where $\tilde{p}_{L}$ and $\tilde{p}_{U}$ are the approximate lower and upper bounds, respectively. This error is computed for all combinations of $n$ and $\hat{p}$. Because the definition of "success" on each Bernoulli trial is arbitrary, we only consider the range $0<\hat{p} \leq \frac{1}{2}$. Figures $1,2,3$, and 4 have mirror images for the range $\frac{1}{2} \leq \hat{p}<1$. The stated coverage is $1-\alpha=0.95$ throughout this section.

Figure 1 contains a plot of $n$ versus $\hat{p}$ for $n=2,4, \ldots, 100$ and considers the

range $0<\hat{p} \leq \frac{1}{2}$ for a maximum absolute error of 0.01 . Thus if the error for a particular $(n, \hat{p})$ pair is greater than 0.01 , the point lands in the middle region. If one of the two approximations yields an error of less than 0.01, then the pair belongs to either the "normal approximation" or "Poisson approximation" region, depending on which yields a smaller error. Not surprisingly, the normal approximation performs better when the point estimate is closer to $\frac{1}{2}$ and the Poisson approximation performs better when the point estimate is closer to 0 . Both approximations perform better as $n$ increases. In order to avoid any spurious discontinuities in the regions, the calculations were made for even values of $n$. The edges of the region are not smooth because of the discrete natures of $n$ and $\hat{p}$. If the horizontal axis were extended, the normal and Poisson regions would meet at approximately $n=150$. Mathematica (Wolfram 1991) was used for the comparisons because of its ability to hold variables to arbitrary precision. 
If the maximum absolute error is relaxed to 0.04 , then there are more cases where the approximations perform adequately. Figure 2 is analogous to Figure 1, but considers an error of 0.04. This figure also contains the rules of thumb associated with the normal and Poisson approximations to the binomial distribution. In particular,

- the rule labeled "R1" is a plot of $\hat{p}=5 / n$ on $[10,100]$ corresponding to the normal approximation rule $n \hat{p} \geq 5$ and $n(1-\hat{p}) \geq 5$

- the rule labeled "R2" is a plot of $\hat{p}=\frac{4}{4+n}$ on $[4,100]$ corresponding to the normal approximation rule $\hat{p} \pm 2 \sqrt{\frac{\hat{p}(1-\hat{p})}{n}}$ falling in the interval $(0,1)$

- the rule labeled "R3" is a plot of $\hat{p}=\frac{9}{9+n}$ on $[9,100]$ corresponding to the normal approximation rule $n>9 \max \left\{\frac{1-\hat{p}}{\hat{p}}, \frac{\hat{p}}{1-\hat{p}}\right\}$

- the rule labeled "R4" is a plot of $\hat{p}=\frac{1}{2}-\frac{\sqrt{n(n-40)}}{2 n}$ on $[40,100]$ corresponding to the normal approximation rule $n \hat{p}(1-\hat{p}) \geq 10$

- the rule labeled "R5" is a plot of $\hat{p}=\frac{1}{2}-\frac{\sqrt{n(n-36)}}{2 n}$ on $[36,100]$ corresponding to the normal approximation rule $n \hat{p}(1-\hat{p})>9$

- the rule labeled "R6" is a plot of $n \geq 20$ and $\hat{p} \leq 0.05$ corresponding to one of the guidelines for using the Poisson approximation.

The $n, \hat{p}$ combinations falling above the dotted curves for rules R1-R5 correspond to those that would be used if the rules of thumb were followed. Clearly, rules R4 and R5 are significantly more conservative than R1-R3 for moderate $n$.

Figure 3 is a continuation of Figure 2 for sample sizes larger than $n=100$. Note that the vertical axis has been modified and the horizontal axis is logarithmic. The curve in the figure represents the values where the maximum absolute errors in using the normal and Poisson approximations are approximately equal. Because this relationship is approximately linear, a rather unwieldy rule of thumb for $n$ between 
100 and 10,000 is: use the normal approximation over the Poisson approximation if $\hat{p}>\frac{5.2-\log _{10} n}{18.8}$.

All of the comparisons thus far have been based on the maximum absolute error. The maximum relative error can be defined by

$$
\max \left\{\left|\frac{p_{L}-\tilde{p}_{L}}{p_{L}}\right|,\left|\frac{p_{U}-\tilde{p}_{U}}{p_{U}}\right|\right\} .
$$

For a maximum relative error of 0.04 , Figure 4 shows a significantly different pattern than that of Figure 2 because the use of the relative error favors the normal approximation over the Poisson.

\section{CONCLUSIONS}

Although there are a number of different variations of the calculations that have been conducted here (e.g., one-sided confidence intervals, different significance levels), there are four general conclusions that would be drawn regardless of the variations considered:

- The traditional advice from most textbooks of using the normal and Poisson approximations to the binomial for the purpose of computing confidence intervals for $p$ should be tempered with a statement such as: "the Poisson approximation should be used when $n \geq 20$ and $p \leq 0.05$ at $\alpha=0.05$ if the analyst can tolerate an absolute error in either limit that may be as large as 0.04" (see Figure 2).

- For sample sizes larger than 150, the maximum absolute error of the upper and lower confidence limit is less than 0.01 if the appropriate approximation technique is used. Figure 3 should be consulted for specific guidance as to whether to use the binomial or Poisson approximation.

- Introductory probability and statistics textbooks targeting statistics and mathematics majors would benefit from including the use of the $F$ distribution to 
find $p_{L}$ and $p_{U}$. Many statistical software packages calculate percentiles of the $F$ distribution. Approximate methods should be used only when the numerical methods associated with exact methods fail.

- More of these texts should include the use of the Poisson approximation to the binomial distribution for determining interval estimates for $p$. These confidence limits only require a table look-up associated with the chi-square distribution and are very accurate for large $n$ and small $p$.

\section{APPENDIX A}

Two derivations of the exact confidence intervals for $p$ that depend on the $F$ distribution are given here. The first was given in Blyth (1986) and the second in Hald (1952).

Let $W_{1}, W_{2}, \ldots, W_{n}$ be iid $\mathrm{U}(0,1)$ random variables. Let $Y$ be the number of the $W_{i}$ 's that are less than $p$. Hence $Y$ is binomial with parameters $n$ and $p$. Using a result from Casella and Berger (1990, p. 223), the order statistic $W \equiv W_{(y)}$ has the beta distribution with parameters $y$ and $n-y+1$. Because the events $Y \geq y$ and $W<p$ are equivalent, $P(Y \geq y)$, which is necessary for determining $p_{L}$, can be calculated by

$$
\begin{aligned}
P(Y \geq y) & =P(W<p) \\
& =\frac{\Gamma(n+1)}{\Gamma(y) \Gamma(n-y+1)} \int_{0}^{p} w^{y-1}(1-w)^{n-y} d w .
\end{aligned}
$$

Using the substitution $t=\frac{(n-y+1) w}{y(1-w)}$ and simplifying yields

$$
\begin{aligned}
P(Y \geq y) & =\frac{\Gamma(n+1)}{\Gamma(y) \Gamma(n-y+1)}\left(\frac{n-y+1}{y}\right)^{n-y+1} \int_{0}^{\frac{(n-y+1) p}{y(1-p)}} \frac{t^{y-1}}{\left(\frac{n-y+1}{y}+t\right)^{n+1}} d t \\
& =P\left(F_{2 y, 2(n-y+1)}<\frac{(n-y+1) p}{y(1-p)}\right) .
\end{aligned}
$$


Because this probability equals $\alpha / 2$ for a two-sided confidence interval,

$$
F_{2 y, 2(n-y+1), 1-\alpha / 2}=\frac{(n-y+1) p_{L}}{y\left(1-p_{L}\right)}
$$

or

$$
p_{L}=\frac{1}{1+\frac{n-y+1}{y F_{2 y, 2(n-y+1), 1-\alpha / 2}}}
$$

where the third subscript on $F$ refers to the right-hand tail probability. In a similar fashion

$$
p_{U}=\frac{1}{1+\frac{n-y}{(y+1) F_{2(y+1), 2(n-y), \alpha / 2}}} .
$$

The second derivation of the exact confidence interval for $p$ uses integration by parts to show that the cumulative distribution function for the binomial distribution can be expressed in terms of percentiles of a random variable having the $F$ distribution. The binomial cumulative distribution function can be rewritten as an integral (Hald 1952)

$$
\sum_{k=0}^{y}\left(\begin{array}{c}
n \\
k
\end{array}\right) p^{k}(1-p)^{n-k}=n\left(\begin{array}{c}
n-1 \\
y
\end{array}\right) \int_{p}^{1} w^{y}(1-w)^{n-y-1} d w .
$$

To prove this identity, we successively use integration by parts. (Details omitted here; the interested reader should see Hald 1952.) Noting that the incomplete beta function is

$$
B_{p}(y+1, n-y)=\int_{0}^{p} w^{y}(1-w)^{n-y-1} d w
$$

and that the complete beta function is

$$
B(y+1, n-y)=\int_{0}^{1} w^{y}(1-w)^{n-y-1} d w
$$

we have from (1)

$$
\sum_{k=0}^{y}\left(\begin{array}{c}
n \\
k
\end{array}\right) p^{k}(1-p)^{n-k}=\frac{B(y+1, n-y)-B_{p}(y+1, n-y)}{B(y+1, n-y)} .
$$


Now let $W$ denote an $F$-distributed random variable with $\left(f_{1}, f_{2}\right)$ degrees of freedom. Then the random variable $Z=\frac{f_{1} W}{f_{2}+f_{1} W}$ can be shown to have the cumulative distribution function (Hald 1952)

$$
F_{Z}(z)=P(Z \leq z)=\frac{B_{z}\left(f_{1} / 2, f_{2} / 2\right)}{B\left(f_{1} / 2, f_{2} / 2\right)} \quad 0<z<1 .
$$

It follows from $(2)$ that for $f_{1}=2(y+1)$ and $f_{2}=2(n-y)$

$$
\begin{aligned}
\sum_{k=0}^{y}\left(\begin{array}{c}
n \\
k
\end{array}\right) p^{k}(1-p)^{n-k} & =1-P(Z \leq p) \\
& =1-P\left(\frac{(y+1) W}{n-y+(y+1) W} \leq p\right) \\
& =1-P\left(W \leq\left(\frac{n-y}{y+1}\right)\left(\frac{p}{1-p}\right)\right) .
\end{aligned}
$$

The lower confidence limit $p_{L}$ satisfies

$$
\sum_{k=y}^{n}\left(\begin{array}{c}
n \\
k
\end{array}\right) p_{L}^{k}\left(1-p_{L}\right)^{n-k}=\alpha / 2,
$$

for $y=1,2, \ldots, n-1$. Equivalently

$$
\sum_{k=0}^{y-1}\left(\begin{array}{c}
n \\
k
\end{array}\right) p_{L}^{k}\left(1-p_{L}\right)^{n-k}=1-\alpha / 2
$$

or

$$
P\left(V \leq\left\{\frac{n-y+1}{y}\right\}\left\{\frac{p_{L}}{1-p_{L}}\right\}\right)=\alpha / 2
$$

where $V \sim \mathrm{F}(2 y, 2(n-y+1))$. To express $p_{L}$ in terms of the percentiles of the $F$ distribution,

$$
\left(\frac{n-y+1}{y}\right)\left(\frac{p_{L}}{1-p_{L}}\right)=F_{2 y, 2(n-y+1), 1-\alpha / 2}
$$

or

$$
p_{L}=\frac{1}{1+\frac{n-y+1}{y F_{2 y, 2(n-y+1), 1-\alpha / 2}}} .
$$

Similarly

$$
p_{U}=\frac{1}{1+\frac{n-y}{(y+1) F_{2(y+1), 2(n-y), \alpha / 2}}} .
$$




\section{APPENDIX B}

The Mathematica code for finding exact confidence intervals for $p$ given $n, y$, and $\alpha$ based on the binomial distribution is

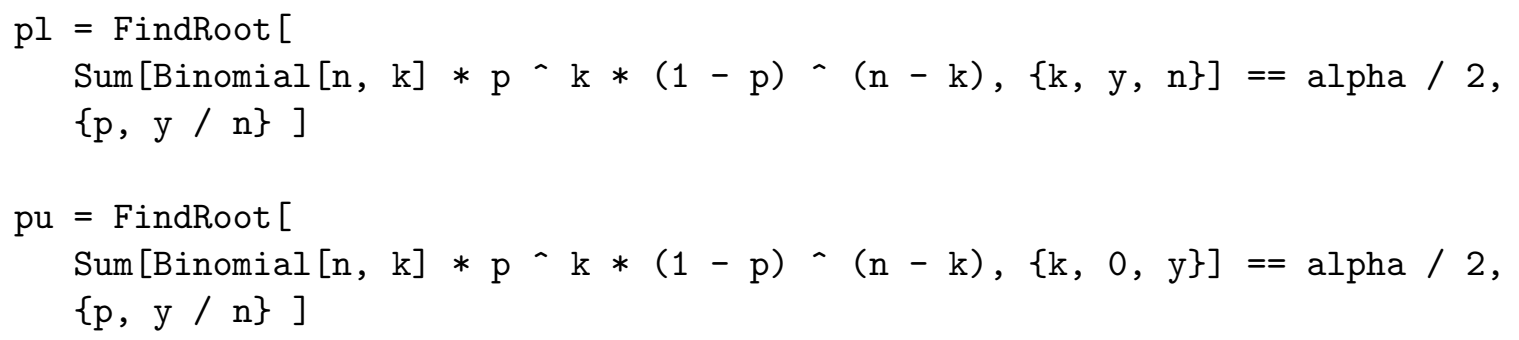

\section{APPENDIX C}

The Mathematica code for finding exact confidence intervals for $p$ given $n, y$, and $\alpha$ based on the $F$ distribution is

<<tatistics 'ContinuousDistributions'

fcrit $=$ Quantile[FRatioDistribution $[2 * \mathrm{y}, 2 *(\mathrm{n}-\mathrm{y}+1)]$, alpha / 2] $\mathrm{pl}=1 /(1+(\mathrm{n}-\mathrm{y}+1) /(\mathrm{y} *$ fcrit $))$

fcrit $=$ Quantile[FRatioDistribution $[2 *(\mathrm{y}+1), 2 *(\mathrm{n}-\mathrm{y})], 1-$ alpha / 2] $\mathrm{pu}=1 /(1+(\mathrm{n}-\mathrm{y}) /((\mathrm{y}+1) *$ fcrit $))$

\section{REFERENCES}

Aczel, A. D. (1993), Complete Business Statistics (2nd ed.), Homewood, IL: Irwin.

Anderson, D. R., Sweeney, D. J., and Williams, T. A. (1994), Introduction to Statistics: Concepts and Applications (3rd ed.), St. Paul, MN: West Publishing.

Azzam, A., Halawa, A., and Hussien, O. (1991), "Choosing between Normal and Poisson Approximations to the Cumulative Binomial Probabilities: A New Method," The Egyptian Statistical Journal, 35, 1, 125-143.

Blyth, C. R. (1986), "Approximate Binomial Confidence Limits," Journal of the American Statistical Association, 81, 843-855; Corrigenda (1989), 84, 636. 
Blyth, C. R., and Still, H. (1983), "Binomial Confidence Intervals," Journal of the American Statistical Association, 78, 108-116.

Casella, G., and Berger, R. (1990), Statistical Inference, Pacific Coast, CA: Wadsworth and Brooks/Cole.

Chen, H. (1990), "The Accuracy of Approximate Intervals for a Binomial Parameter," Journal of the American Statistical Association, 85, 514-518.

Clopper, C. J., and Pearson, E. S. (1934), "The Use of Confidence or Fiducial Limits Illustrated in the Case of the Binomial," Biometrika, 26, 404-413.

Copas, J. B. (1992), "Exact Confidence Limits for Binomial Proportions - Brenner \& Quan Revisited," The Statistician, 41, 569-572.

Creighton, J. H. C. (1994), A First Course in Probability Models and Statistical Inference, New York: Springer-Verlag.

Daly, L. (1992), "Simple SAS Macros for the Calculation of Exact Binomial and Poisson Confidence Limits," Computers in Biology and Medicine, 22, 351-361.

Freund, J. E. (1992), Mathematical Statistics (5th ed.), Englewood Cliffs, NJ: PrenticeHall.

Ghosh, B. K. (1979), "A Comparison of Some Approximate Confidence Intervals for the Binomial Parameter," Journal of the American Statistical Association, 74, 894-900.

Goldman, R. N., and Weinberg, J. S. (1985), Statistics: An Introduction, Englewood Cliffs, NJ: Prentice-Hall.

Hald, A. (1952), Statistical Theory with Engineering Applications, New York: John Wiley. 
Hogg, R. V., and Tanis, E. A. (1993), Probability and Statistical Inference (4th ed.), New York: Macmillan.

Jarrell, S. B. (1994), Basic Statistics, Dubuque, IA: William C. Brown.

Kelly, D. G. (1994), Introduction to Probability, New York: Macmillan.

Lapin, L. L. (1990), Probability and Statistics for Modern Engineering (2nd ed.), Boston: PWS-KENT.

Larsen, R. J., and Marx, M. L. (1986), An Introduction to Mathematical Statistics and Its Applications (2nd ed.), Englewood Cliffs, NJ: Prentice-Hall.

Larson, H. J. (1995), Introduction to Probability, Reading, MA: Addison-Wesley.

Lehmann, E. L., and Loh, W. (1990), "Pointwise Versus Uniform Robustness of Some Large-sample Tests and Confidence Intervals," Scandanavian Journal of Statistics, 17, 177-187.

Lentner, C. (ed.) (1982), Giegy Scientific Tables (Vol. 2), Basel, Switzerland: CibaGeigy.

Ling, R. F. (1992), "Just Say No to Binomial (and other Discrete Distributions) Tables," The American Statistician, 46, 53-54.

Mason, R. D., Lind, D. A., and Marchal, W. G. (1994), Statistics: An Introduction (4th ed.), Fort Worth, TX: Harcourt, Brace \& Company.

Mendenhall, W., and Sincich, T. (1992), Statistics for Engineering and the Sciences (3rd ed.), New York: Macmillan.

Poor, H. V. (1991), "The Maximum Difference Between the Binomial and Poisson Distributions," Statistics \& Probability Letters, 11, 103-106. 
Ross, S. (1994), A First Course in Probability (4th ed.), New York: Macmillan.

Schader, M., and Schmid, F. (1989), "Two Rules of Thumb for the Approximation of the Binomial Distribution by the Normal Distribution," The American Statistician, 43, 23-24.

Scheaffer, R. L., and McClave, J. T. (1990), Probability and Statistics for Engineers (3rd ed.), Boston: PWS-Kent.

Triola, M. F. (1995), Elementary Statistics (5th ed.), Reading, MA: Addison-Wesley.

Trivedi, K. S. (1982), Probability \& Statistics with Reliability, Queuing and Computer Science Applications, Englewood Cliffs, NJ: Prentice-Hall.

Vollset, S. E. (1993), "Confidence Intervals for a Binomial Proportion," Statistics in Medicine, 12, 809-824.

Walpole, R. E., and Myers, R. H. (1993), Probability and Statistics for Engineers and Scientists (5th ed.), New York: Macmillan.

Weiss, N. A. (1995), Introductory Statistics (4th ed.), Reading, MA: Addison-Wesley.

Wolfram, S. (1991), Mathematica: A System for Doing Mathematics by Computer (2nd ed.), Redwood City, CA: Addison-Wesley. 


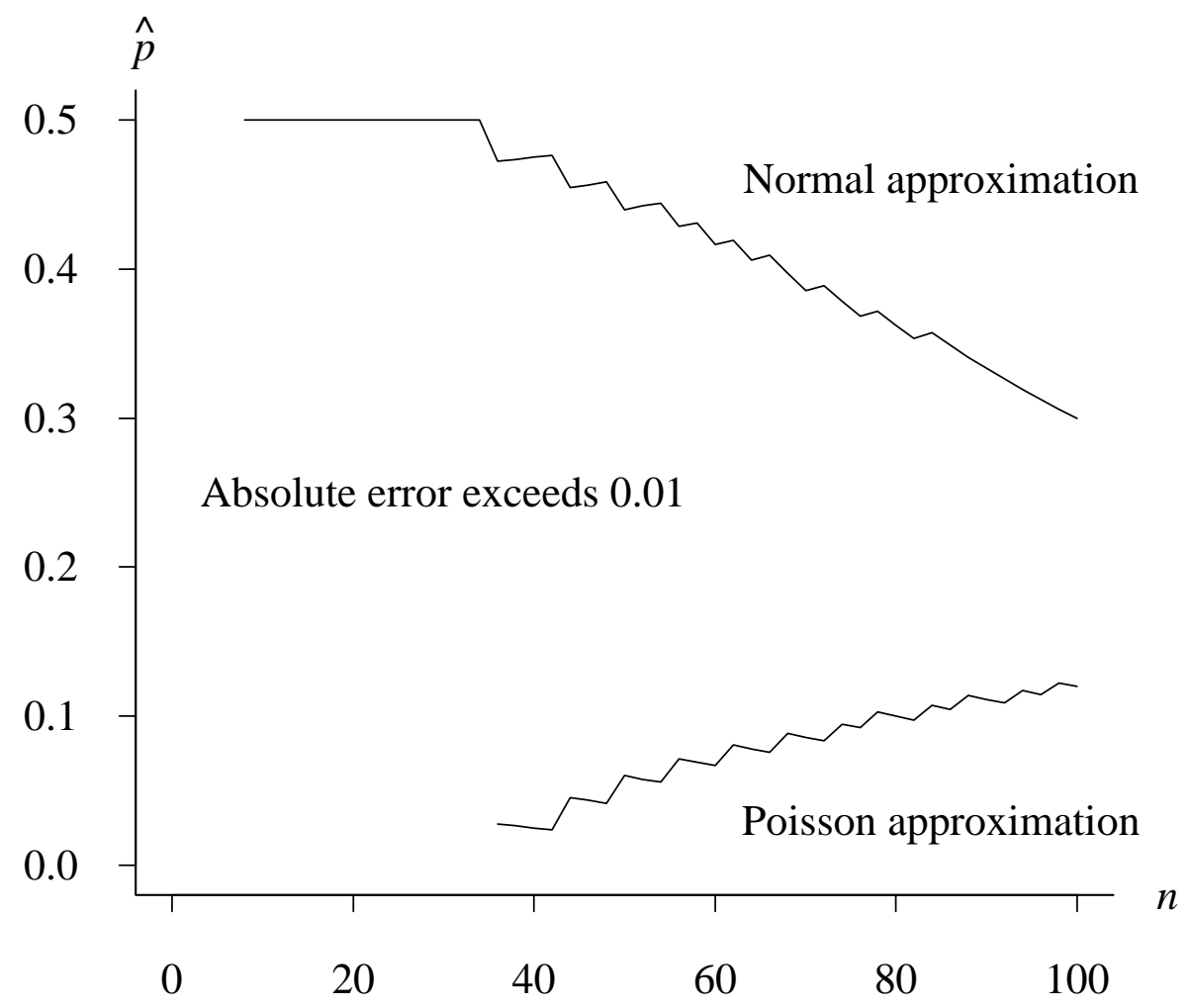

Figure 1: Approximation methods for confidence limits (maximum absolute error: $0.01)$. 


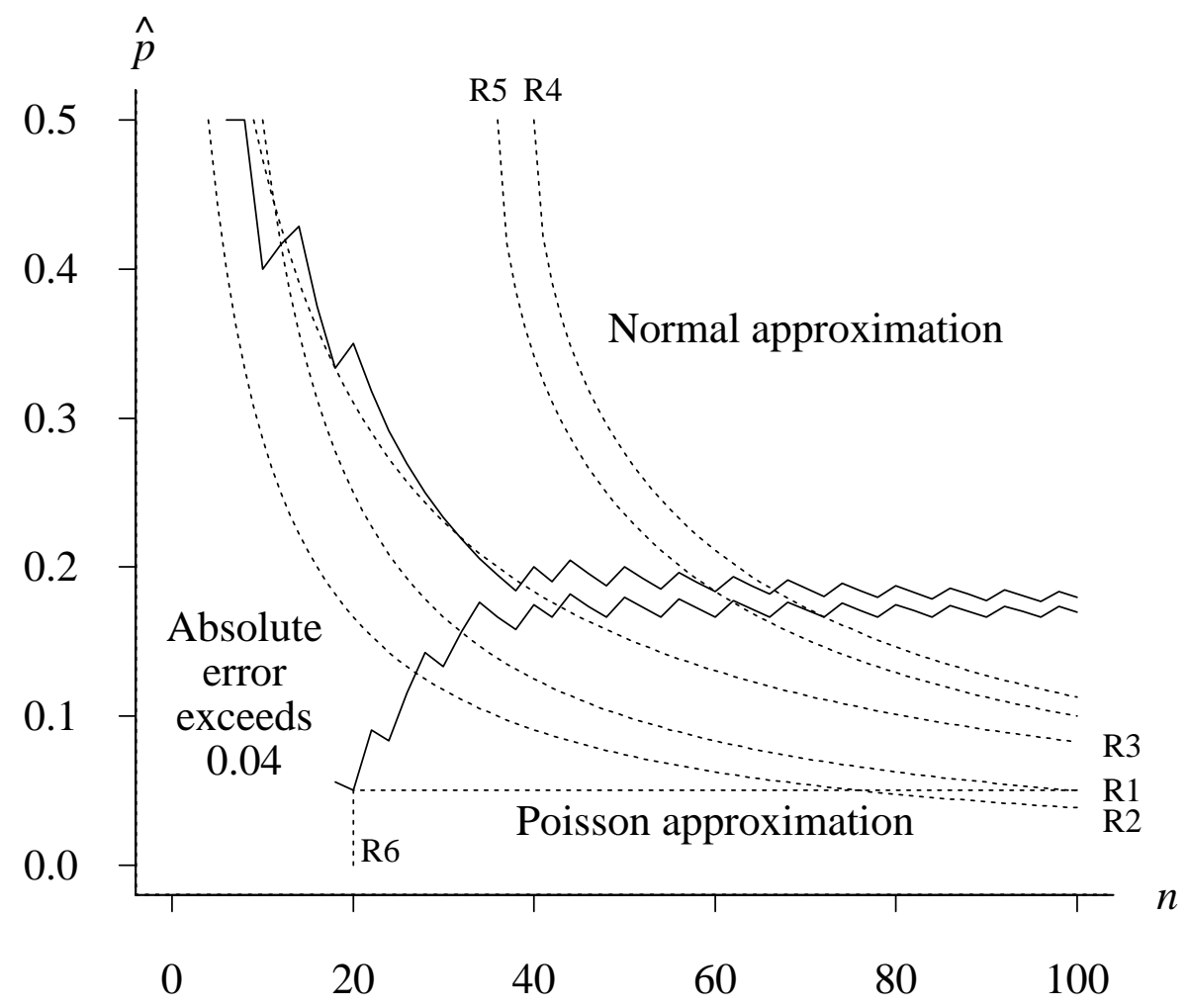

Figure 2: Approximation methods for confidence limits (maximum absolute error: 0.04 ) and rules of thumb. 


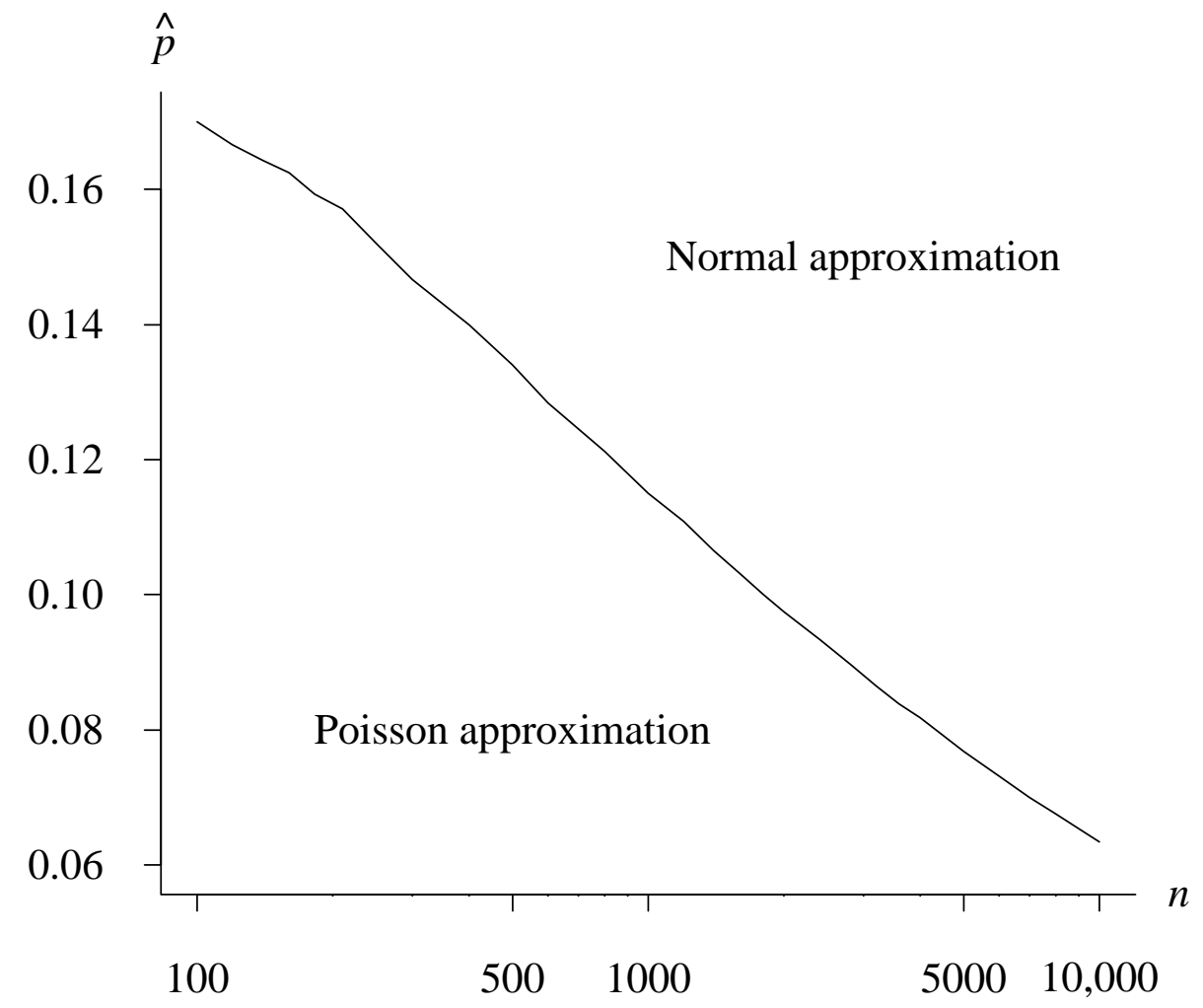

Figure 3: Poisson vs. normal approximations for large sample sizes. 


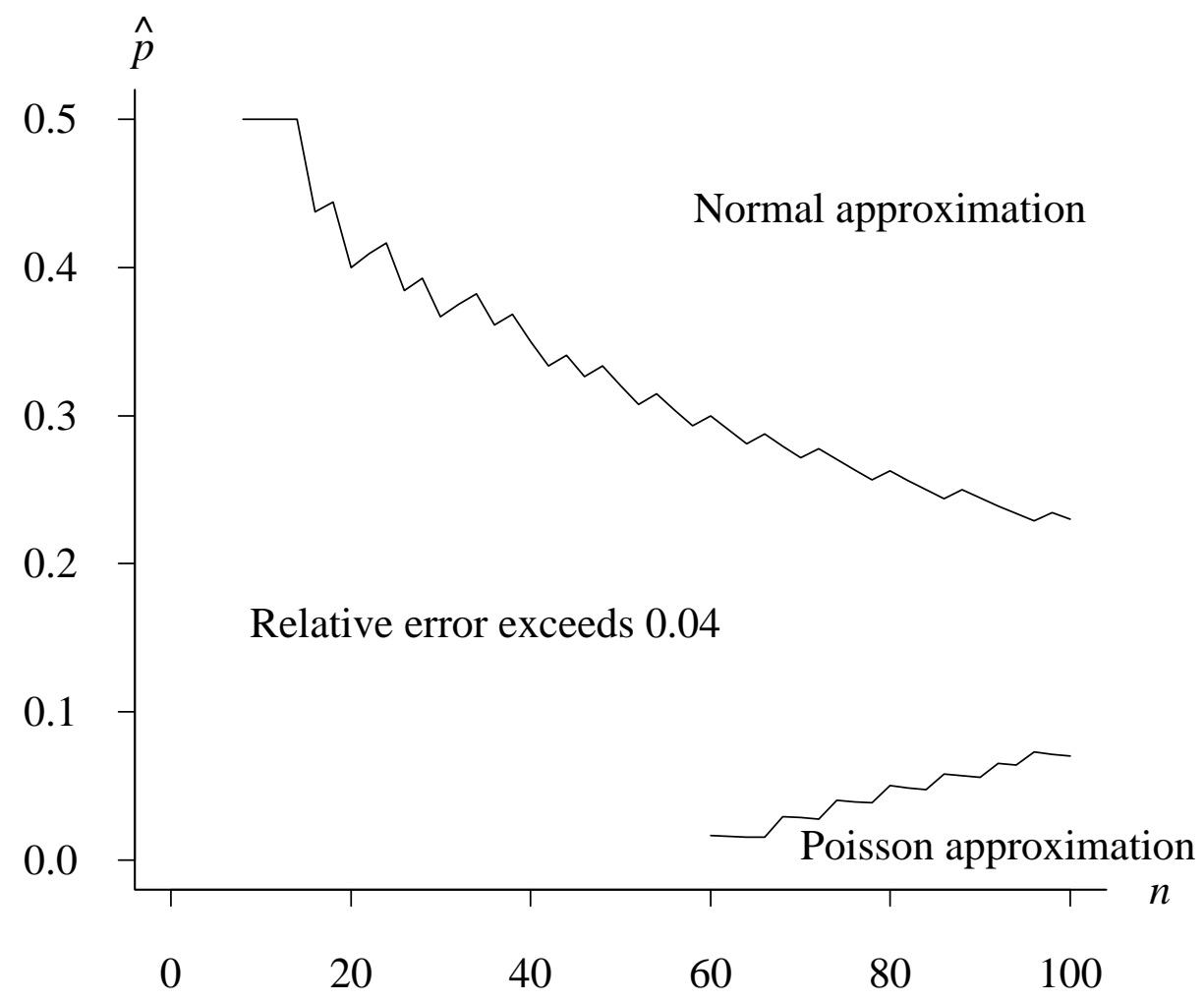

Figure 4: Approximation methods for confidence limits (relative maximum error: $0.04)$. 